\title{
Automated insertion of package dies onto wire and into a textile yarn sheath
}

\author{
Dorothy Anne Hardy ${ }^{1}$ (D) Ioannis Anastasopoulos ${ }^{1} \cdot$ Mohamad-Nour Nashed $^{1}$ (I) $\cdot$ Carlos Oliveira ${ }^{1} \cdot$

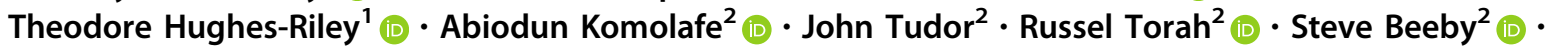 \\ Tilak Dias ${ }^{1}$ (])
}

Received: 30 August 2018 / Accepted: 19 February 2019/Published online: 4 March 2019

(C) The Author(s) 2019

\begin{abstract}
Wider adoption of electronic textiles requires integration of small electronic components into textile fabrics, without comprising the textile qualities. A solution is to create a flexible yarn that incorporates electronic components within the fibres of the yarn (E-yarn). The production of these novel E-yarns was initially a craft skill, with the inclusion of package dies within the fibres of the yarn taking about $90 \mathrm{~min}$. The research described here demonstrated that it is possible to produce E-yarns on an industrial scale by automating the manufacturing process. This involved adapting printed circuit board manufacturing technology and textile yarn covering machinery. The production process started with re-flow soldering of package dies onto fine multi-strand copper wire. A carrier yarn was then placed in parallel with the copper wire to provide tensile strength. The package die and adjacent carrier yarn were then encapsulated in a polymer micro-pod to provide protection from moisture ingress and from mechanical strain on the die and solder joints. The process was then completed by surrounding the micro-pod and copper interconnects with additional fibres, held tightly together with a knitted fibre-sheath. This prototype, automated production process reduced the time for embedding one micro-device within a yarn to $6 \mathrm{~min}$, thus increasing the production speed, demonstrating that automation of the E-yarn production process is feasible. Prototype garments have been created using E- yarns. Further developments can include automated transfer of the yarn components from one stage of production to the next, enabling greater increases in speed of manufacture of E yarns.
\end{abstract}

\section{Introduction}

The growing market for electronic textiles is predicted to be 'approaching \$5bn by 2027' (Hayward 2017) showing that there is a desire to place electronics within clothing (Tomico et al. 2017). Satisfying this market for electronic textiles and wearables will require the integration of electronics into fabrics, whilst maintaining the ability of the textiles to drape and conform to the body. Initial developments in electronic textiles included electronic devices within pockets, or attached to garments. Subsequently,

Dorothy Anne Hardy

dorothy.hardy@ntu.ac.uk

1 Advanced Textiles Research Group, Nottingham Trent University, Bonington Building, Dryden Street, Nottingham NG1 4GG, UK

2 Electronics and Computer Science, University of Southampton, University Road, Southampton SO17 1BJ, UK electronic textiles were developed with electronic functionality added by incorporating conductive yarns into a textile (Hughes-Riley et al. 2018). Further developments led to integration of electronics into textiles through incorporation of small electronic components such as multi-terminal package dies within the yarn structure (Dias 2005, 2016). These electronic yarns (E-yarns) have been developed over the course of 13 years.

The basic concept is to encapsulate semiconductor microchips within polymer micro-pods, in order to protect them from the mechanical and chemical stresses that the E-yarns would be subjected to during conventional textile fabric and garment manufacture, and the day-to-day use of textiles incorporated within garments. The micro-pods and interconnects, made of fine conductive fibres/wires, are then integrated within the fibres of a textile yarn, in a manner such that the interconnects are positioned co-axially in the yarn structure. This fibre assembly ensures that the electronics are hidden and not visible to the naked eye. 
The use of micro-pods enables the E-yarns to be washed. These functionalities are not available with earlier types of E-textile, and are of immense importance for consumer acceptance of E-textiles. The resulting E-yarns can be incorporated into electronic textiles using conventional textile manufacturing processes such as knitting, weaving or embroidery. The challenge is to ensure that the electronics take up minimal space, and that the entire yarn construction is as flexible as possible, so that the components can then be included within a textile construction that can shear, drape and conform. The novelty of the E-yarn technology is in the protection of the semi-conductor die within a micro-pod, in order to embed the die within the fibres of a textile yarn, and the use of a miniature circular knitting process in which the copper wire interconnects with micro-pods are placed co-axially within a yarn structure. This prevents migration of the copper wire onto the surface of the yarn and renders the electronics invisible to the naked eye.

The proof-of-concept of E-yarn production was initially a time-consuming craft skill (Rathnayake 2015), with manufacture of each section of E-yarn containing a sensor or LED taking 60-90 min. Prototype demonstrators were manufactured with E-yarns made using this manual production process. These include temperature-sensing wound dressings (Lugoda et al. 2018); helmet covers containing acoustic-sensing yarn (Hughes-Riley and Dias 2018a); and a vibration-sensing glove, for monitoring of vibrations transmitted to the hand by power tools (Hughes-Riley and Dias 2018b). The increasing industrial interest in E-yarn meant that automation of the E-yarn production process was required so that E-yarns could be manufactured on a scale compatible with the demand.

The aim of the work described here is to develop a working prototype process for the manufacture of E-yarns. This could then be scaled up by industry. The objective of the development was to demonstrate the feasibility of E-yarn production at commercially acceptable quantities. It is envisaged that later iterations of the design will bring the manufacturing cost down. The goal was to automate the process within a 6-month timeframe, so that the production speed of E-yarns could be increased. The details below are for the manufacture of an LED-yarn. Other, small package dies can also be incorporated into E-yarn using the method described. This paper is a development of a conference paper An Automated Process for Inclusion of Package Dies and Circuitry within a Textile Yarn (Hardy et al. 2018b).

\subsection{E-yarn materials and construction}

One of the key features of E-yarns is their flexibility and washability. The flexibility is due to the use of polymer micro-pods, generally of the order of $3 \mathrm{~mm}^{3}$ volume. These encapsulate semiconductor dies and fine multi-strand copper wire, with a total diameter of $140 \mu \mathrm{m}$, that forms the interconnects. Multi-strand wire provides more flexibility than a single-strand copper wire of the same diameter. Attaching a strong carrier yarn during formation of the micro-pod minimises mechanical stress and strain to the copper wire and solder joints during further processing. The outer, knitted sheath surrounding the wire, carrier yarn and micro-pods enables post-processing of E-yarn on weaving, knitting and embroidery machines. A schematic of an E-yarn is shown in Fig. 1.

\subsection{The craft process of E-yarn manufacture}

The first step in constructing E-yarns was to design a manual process that would enable production in small quantities. Successful development of the process required a combination of yarn handling techniques in addition to precise assembly of the electronic components. Standard electronic circuits are often built-up on printed circuit boards (PCBs) (Li et al. 2004; LaDou 2006), including flexible PCBs (Siegel et al. 2010). PCBs provide a stable platform for attachment of dies and interconnects. The process of manufacturing E-yarns is somewhat similar to that of producing a PCB, however without a firm substrate on which soldering can take place. This, and the need to combine textile fibres and semiconductor package dies, required the design of a new concept. The soldering of fine copper wire to the solder pads of the semiconductor package dies, and the application of resin to form the micro-pod were carried out manually. The stages in the craft production process are described below and shown in Figs. 2, 3 and 4. Details in the diagrams are not to scale.

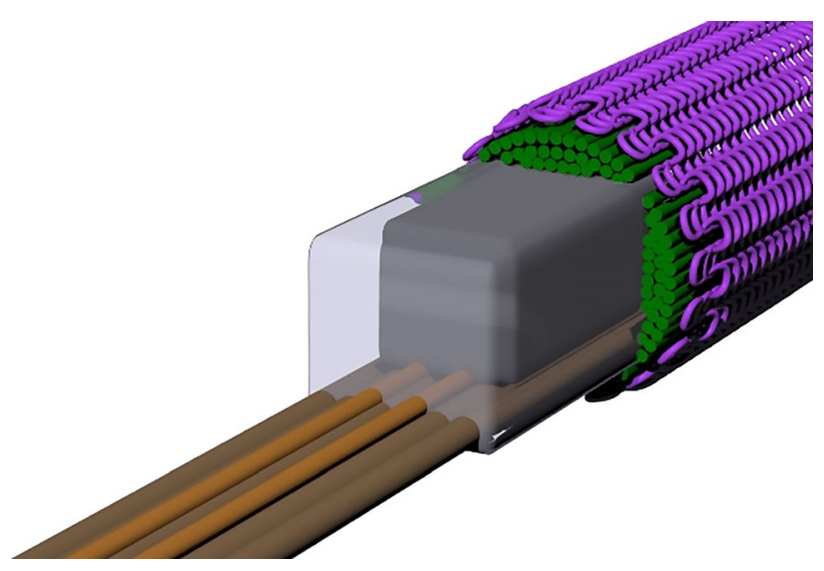

Fig. 1 A schematic of an LED-yarn showing a packaged die surrounded by a micro-pod, with attached copper wire and carrier yarn. A knitted sheath surrounds the construction 


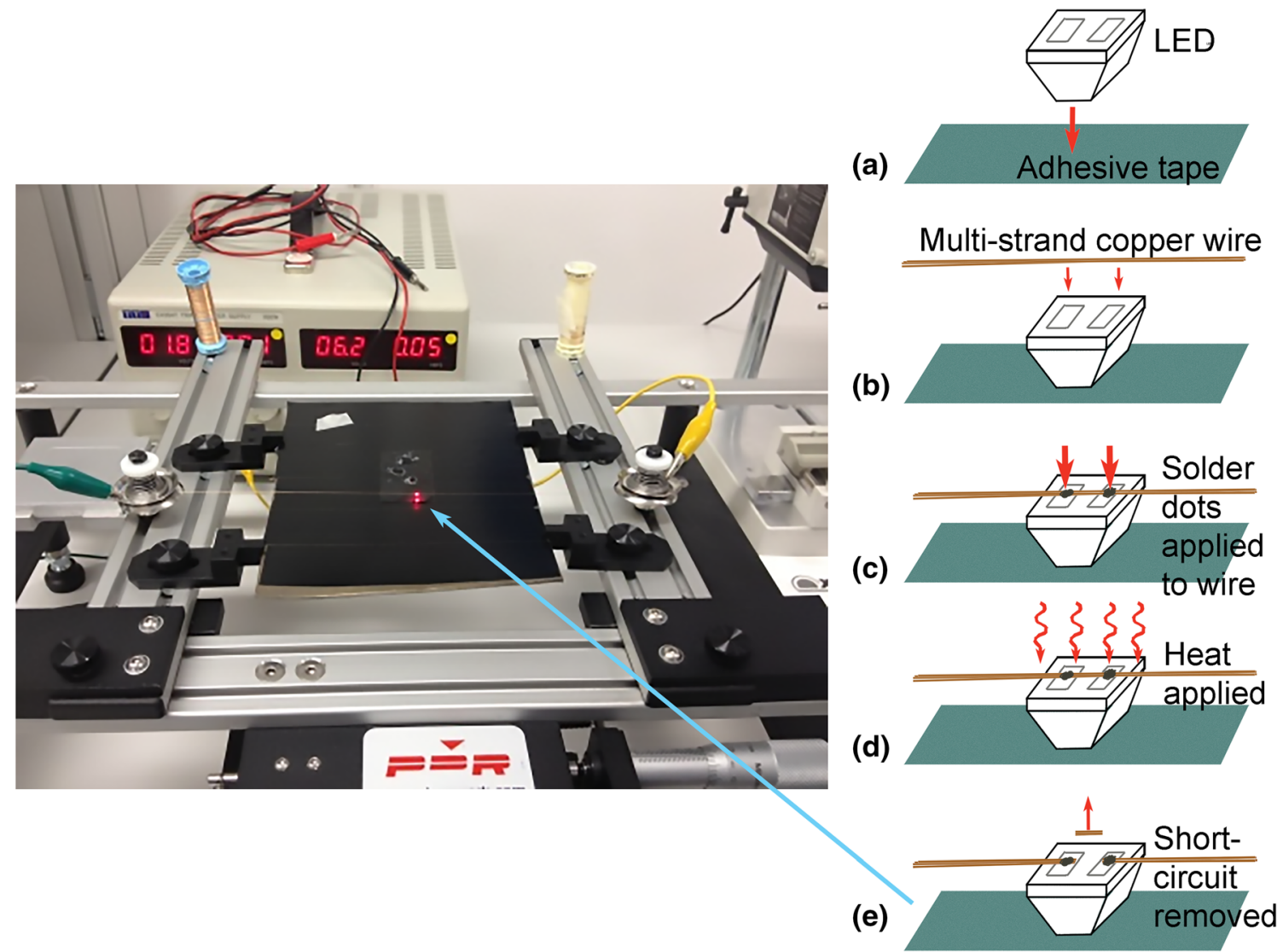

(a)

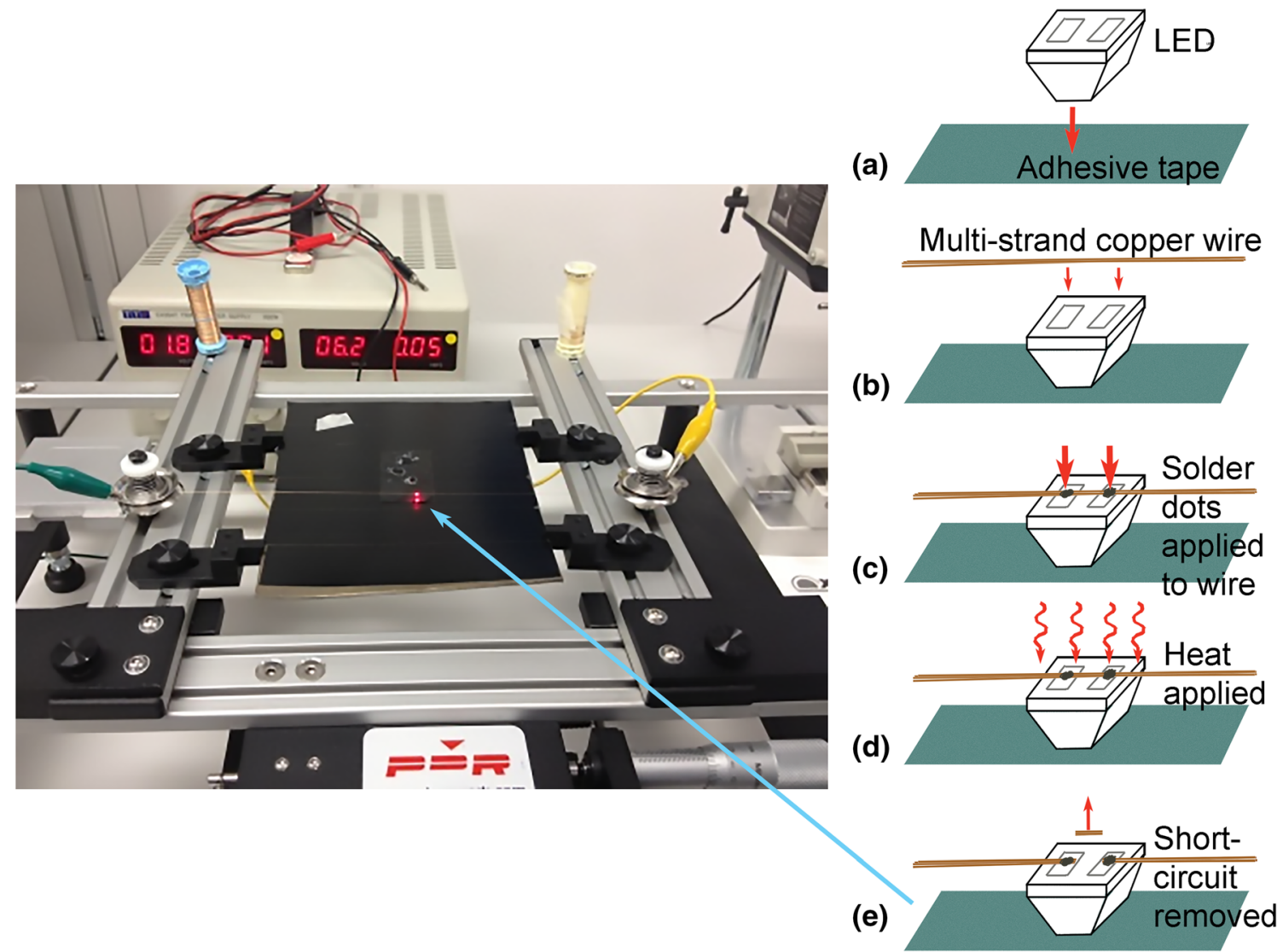

ED

Fig. 2 The bespoke soldering rig used in the manual soldering process, showing an illuminated LED soldered to copper wire (on the left). Diagrams a-e on the right show the steps in soldering the LED onto wire

\subsubsection{Soldering}

The setup for soldering an LED onto copper wire is shown on the left in Fig. 2. The diagrams on the right of Fig. 2 show the stages in the soldering process:

a. A semiconductor package die (Kingbright KPHHS1005SURCK Red LED, 630 nm 1005 (0402), Rectangle Lens SMD package: RS Components, Corby, UK) was placed on a double-sided adhesive tape, with the solder pads facing upwards.

b. A copper wire (7-strand copper wire with $50 \mu \mathrm{m}$ strand diameter: Knight Wire, Potters Bar, UK) was placed over the die; tensioning of copper wire during the process was carried out using a dedicated soldering rig designed for a focused IR reflow soldering station from PDR (XT5P IR Rework Station: PDR, Crawley, UK) shown in Fig. 2.

c. Two dots of solder (lead-free, antimony-free rosinbased solder paste, part number 7024454: Nordson EFD, Dunstable, UK) were dispensed from a pneumatic dispenser (Nordson EFD Ultimus I precision fluid dispenser: Nordson EFD, Dunstable, UK) over the wire that was placed above the die solder pads.

d. Heat was applied from the infra-red (IR) lamp in the PDR system to melt the solder paste, forming two soldered joints.

e. The wire that formed a short circuit between the solder pads was removed manually using a sharp blade.

\subsubsection{Micro-pod formation}

This was achieved with an encapsulation rig shown on the left in Fig. 3. The steps involved are shown in the diagram on the right of Fig. 3. These were:

a. Application of resin (Dymax Multi-cure ${ }^{\circledR}$ 9001-E-V3.5 Resilient: Intertronics, Kidlington, UK) with a handheld syringe.

b. Curing of the resin using a BlueWave 50 UV Light Curing Spot Lamp (Dymax, Torrington, Connecticut, USA). The light guide is the black, curved tube in Fig. 3, with the end directed over the white E-yarn held above the patch of UV light. 


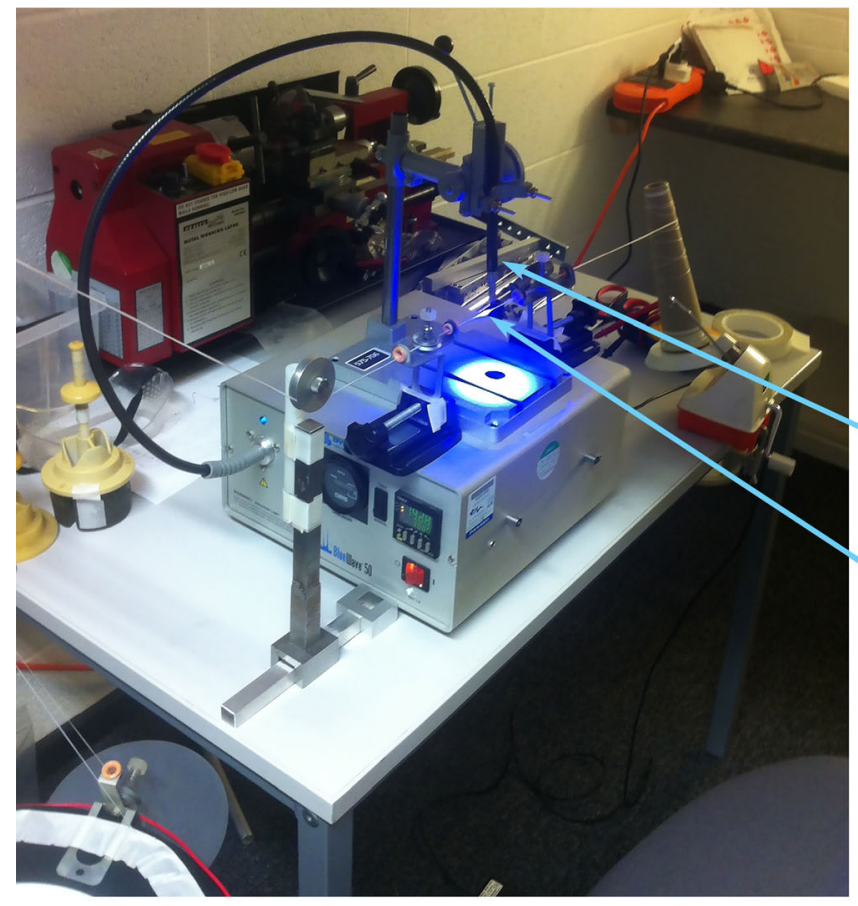

(a)

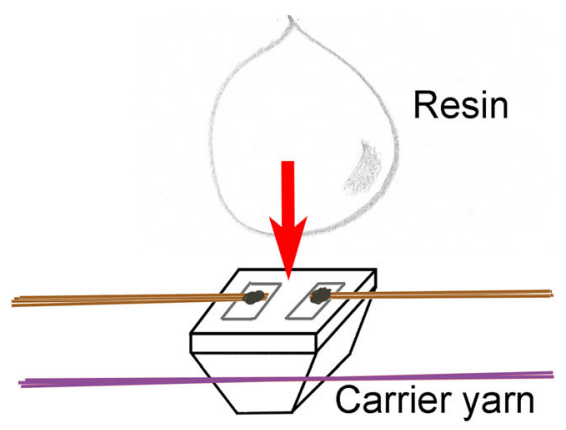

(b)

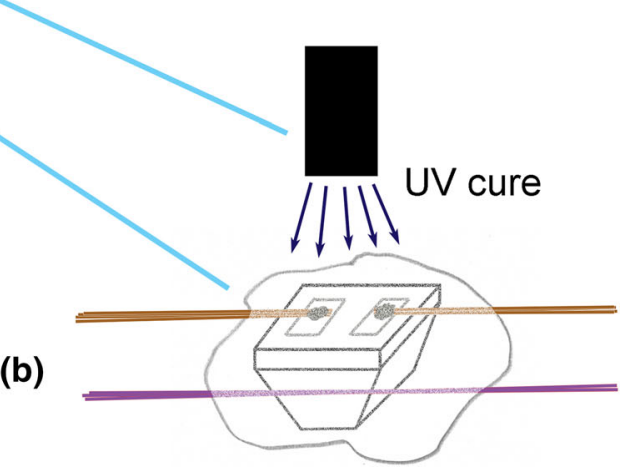

Fig. 3 The encapsulation rig used in the manual process of the formation of a micro-pod around a die (indicated by the lower arrow) is shown on the left. The black light guide (indicated by the top arrow) directed UV light over a partially-formed E-yarn that was held

\subsubsection{Insertion into a textile sheath}

The wire and carrier yarn, with attached micro-pods, were passed through an MC-D small-diameter warp-knitting machine (RIUS, Barcelona, Spain), shown in Fig. 4a. This created a knitted sheath using polyester yarns (167/36: J. H. Ashworth and Son Ltd, Hyde, UK). Figure 4b shows the production of a completed E-yarn in diagrammatic form.

The manual process was time consuming due to the need for accurate manual positioning of small dies such as the 0402 package LEDs (dimensions $1 \times 0.5 \times 0.5 \mathrm{~mm}$ ) on flexible wire, by hand, combined with manual application of solder paste and resin. This manufacturing process required improvement to enable E-yarns to be manufactured more quickly, so that further prototypes could be created in an acceptable time frame.

\section{Methods}

Moving from the manual method to a semi-automated process of E-yarn production required further development of the methods and machinery used in the manual process. The first two stages of the manual process, soldering and encapsulation, were automated separately with a longerterm aim of bringing the processes together to create one, between a pulley on the left and a yarn cone on the right in the photo. The diagrams on the right of the figure show: a a drop of resin positioned to cover the soldered die; b the UV-curing process

continuous production line for E-yarn. An intermediate stage was added to the production process, in which textile yarns were wrapped around the copper wire that formed the conductive core of the E-yarn, before the E-yarn core was inserted into a knitted sheath. This prevented the copper wire core from protruding through the knitted, outer sheath of the E-yarn, which could happen when the E-yarn was flexed. Once the E-yarn production process was automated, tests were carried out to validate the accuracy of LED placement within an E-yarn, and to ascertain the tensile strength of the E-yarn and its components to confirm its suitability for further processing into a textile.

\subsection{Automation of the E-yarn manufacturing process}

An iterative design process was used (Lu and Liu 2012) to examine each of the stages of production and to determine a method to automate each process in a way that could be achieved within the project time and budgetary constraints. Groups of machinery were modified to carry out each stage of the process. The stages of manufacture for an E-yarn containing an LED (Kingbright KPHHS-1005SURCK Red LED, $630 \mathrm{~nm} 1005$ (0402), Rectangle Lens SMD package: RS components, Corby, UK) are described below, but the 


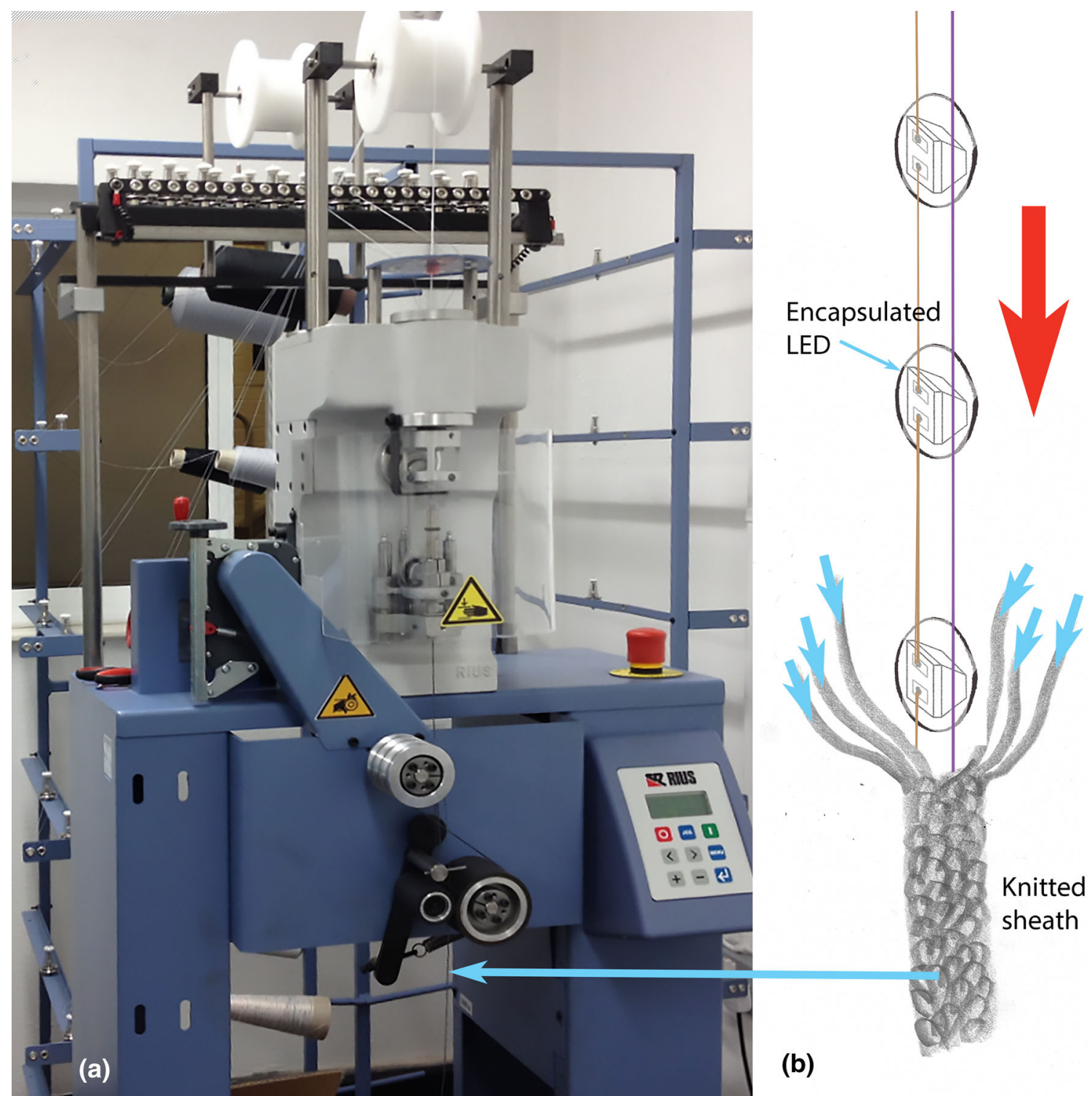

Fig. 4 a The RIUS machine used for the creation of electronic yarn that can be seen emerging from the bottom of the machine, indicated by the long blue arrow; b diagram showing a knitted sheath being formed around an electronic yarn core containing LEDs within micro-pods

process can be applied to other package dies such as thermistors.

\subsubsection{Soldering of semiconductor package dies to wire}

Soldering of semiconductor dies to copper wire required adaption of techniques used in the manufacture of circuitry on PCBs, where dies are positioned onto the conductive tracks on the PCBs. A method was developed to solder dies directly onto wire without the PCB substrate. Automation of die placement on copper wire was required, to ensure accurate, quick placement. The automated setup is shown in Fig. 5. A pick-and-place machine (QM1100: SMT Max, Chino California, USA) was programmed to remove dies from packaging reels and place them with the solder pads in direct contact with the copper wire. The manual method described in Sect. 1.2.1 required dies to be placed with solder pads facing upwards, so that wire could be placed over the dies before applying solder paste onto the solder pads. The new process required adaption for dies placed by the pick-and-place machine, with solder pads facing downward. Reversing the die orientation made it necessary to deposit solder paste onto the wire before placing the die onto the wire, so that the solder paste was sandwiched between the wire and the solder pads on the die. Accurate placement of small amounts of solder (on the order of $0.25 \mathrm{~mm}^{3}$ ) was required. The Nordson EFD equipment (Nordson EFD Ultimus I precision fluid dispenser: 


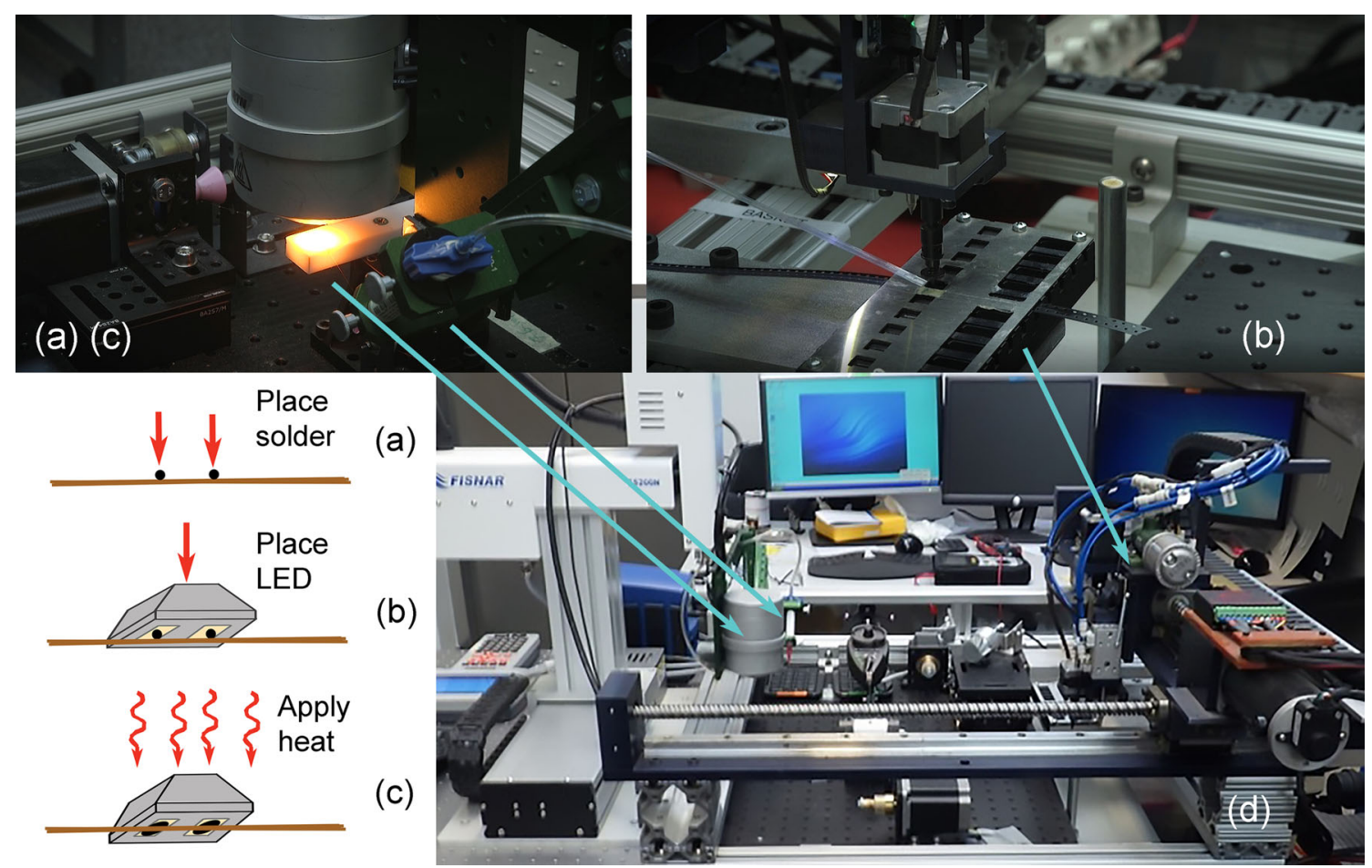

Fig. 5 The automated process of soldering LEDs onto copper wire, with arrows indicating key pieces of machinery. a Application of two solder dots to wire carried out with the solder dispenser attached to

Nordson EFD, Dunstable, UK) could dispense the required volumes of solder paste: however, a method was required to position the dispensing nozzle over the wire to dispense solder dots to align with the solder pads on the die, $0.7 \mathrm{~mm}$ apart. A robot was chosen (Fisnar model F5200N: Intertronics, Kidlington, UK) due to the accuracy with which it could move the solder paste dispensing nozzle (with a resolution of $1 \mu \mathrm{m}$ ). This process required the copper wire to be held at a pre-defined tension, in place, so that solder could be dispensed onto it.

After soldering a die onto the copper wire, it was necessary to move the copper wire, so that further accurate placement and soldering of dies could be carried out. Development of the copper wire delivery system was key to the successful design of this process. The wire delivery system consists of a stepper motor (Vexta 2-phase model PK268-01A: Oriental Motor Co. Ltd., Tokyo, Japan) to advance the wire and a BTSR ultra-feeder unit (BTSR, Olgiata Olona, Italy) to maintain a pre-determined wire tension. As a point-of-information the BTSR Ultra-feeder units are designed to deliver yarn at constant tension at speeds around $5 \mathrm{~ms}^{-1}$ on high production circular knitting machines. The two units were programmed with LabVIEW (LabVIEW 2014: National Instruments, Newbury, UK) to operate in synchronisation.

An IR soldering system (ATN LBS-G400, Berlin, Germany) was employed for application of heat in the the robot; $\mathbf{b}$ placement of LED on wire carried out by the pick-andplace machine; $\mathbf{c}$ application of infra-red heat over the LED carried out by the infra-red lamp; $\mathbf{d}$ an overview of the soldering setup

soldering process: This was attached to the Fisnar robot, so that it could be placed accurately over the die to focus the heat in the required position. Once the soldering process was completed, the wire with the attached die was moved. The wire short circuit between the solder pads of a chip was removed later with a cutting tool, manually; it was envisaged that this would be integrated with the soldering process at a later stage.

\subsubsection{Forming a micro-pod around the semiconductor package die}

In order to automate the process of forming a resin micropod, a method was required to place a measured quantity of resin around the soldered die. At the same time, a strong carrier yarn (Vectran $^{\mathrm{TM}}$ multifilament yarn: Kuraray, Tokyo, Japan) was to be placed alongside the die, to be included within the resin during curing. Placing the wire with the attached die, and the carrier yarn, within a tubular mould, then injecting resin into the mould, gave a method of ensuring that all of the components within the micro-pod were held together. A Preeflow eco-PEN (Intertronics, Kidlington, Oxfordshire, UK) was used to apply measured doses of resin (Dymax Multi-cure ${ }^{\circledR}$ 9001-E-V3.5 Resilient: Intertronics, Kidlington, UK) into a tubular, silicone-rubber mould. The resin could be UV-cured rapidly through the side of the mould using a Bluewave ${ }^{\circledR} \mathrm{QX} 4{ }^{\mathrm{TM}} \mathrm{UV}$-curing 
system (Intertronics, Kidlington, UK). This system provided a narrow-spectrum light source centred at $385 \mathrm{~nm}$, giving more rapid curing than the wider-spectrum mercury bulb UV source used to for resin curing in the craft method of E-yarn production. A stepper motor (ISM 7411; National Instruments Corporation (U.K.) Ltd., Newbury, UK) was used to pull the carrier yarn in order to extract the micro-pod from the mould. A LabVIEW programme was developed for the sequential triggering of resin injector, UV curing system and the stepper motor (Fig. 6a shows the machinery used to form the micro-pod, with the process shown in diagrammatic form in Fig. 6b).

\subsubsection{Wrapping yarn around the wire and carrier fibre}

This stage of manufacture was added during development of the automated production process to ensure that the copper wire remained within the centre of the completed E-yarn. Twisting textile yarns around the copper wire and carrier yarn ensured that the copper wire could not easily protrude through the exterior of the knitted sheath that formed the outer casing of the E-yarn. A textile yarn processing machine was required to twist textile yarns around the E-yarn core components (copper wire, Vectran ${ }^{\mathrm{TM}}$ and the attached micro-pods). An Agteks DirecTwist 2B6 machine (Agteks, Istanbul, Turkey) (shown in Fig. 7a) was modified to perform this task. The E-yarn core was run through a tensioner (B709014-A: Yuasa Yarn Guide Engineering Co. Ltd., Nagoya, Japan) attached to the exterior casing of the machine, and then through the centre top machine guide. Three strands of cotton yarn (NM
30/1*2 Davidoff: Boyar Textile, Istanbul, Turkey) were guided from the base of the machine into the twisting mechanism, where they were twisted around the E-yarn core (shown in diagrammatic form in Fig. 7b). This machine was designed for use with standard textile yarns, so a slow feed and speed of $10 \mathrm{~m} \mathrm{~min}^{-1}$ was chosen so that the Vectran ${ }^{\mathrm{TM}}$ and copper wire with attached micro-pods could be fed through without being damaged.

\subsubsection{Formation of a knitted sheath around the E-yarn}

The craft method of E-yarn production utilised a RIUS MCD small-diameter warp-knitting machine (Fig. 4) that was used to form a knitted sheath around the core E-yarn materials. The structure was inclined to form a flat tube with bulges where the micro-pods were located. This was changed to a cylindrical yarn construction by addition of packing fibres within the knitted sheath during the knitting process. The process is shown in Fig. 8c. Four polyester yarns (48 f/167 dtex polyester yarns: J. H. Ashworth and Son Ltd, Hyde, UK) were added around the construction. These were fed through a 2-mm diameter cylinder in the machine (Fig. 8b), along with the E-yarn core with surrounding, twisted textile yarns. The knitted sheath was formed around this by a set of six latch needles through which another set of polyester yarns ( $36 \mathrm{f} / 167$ dtex: J. H. Ashworth and Son Ltd, Hyde, UK) were fed. This method of manufacture had previously been found to create a more stable construction than one in which textile yarns were twisted around the central core. Yarn twisting created a structure in which the core was visible on the surface at some points along the yarn. It was
Fig. 6 The machinery used for the creation of a resin micro-pod around the package die, with diagrams showing stages in the process: a injection of resin into a tubular mould containing the LED; $\mathbf{b}$ UV-curing of the resin using the UV light-guide

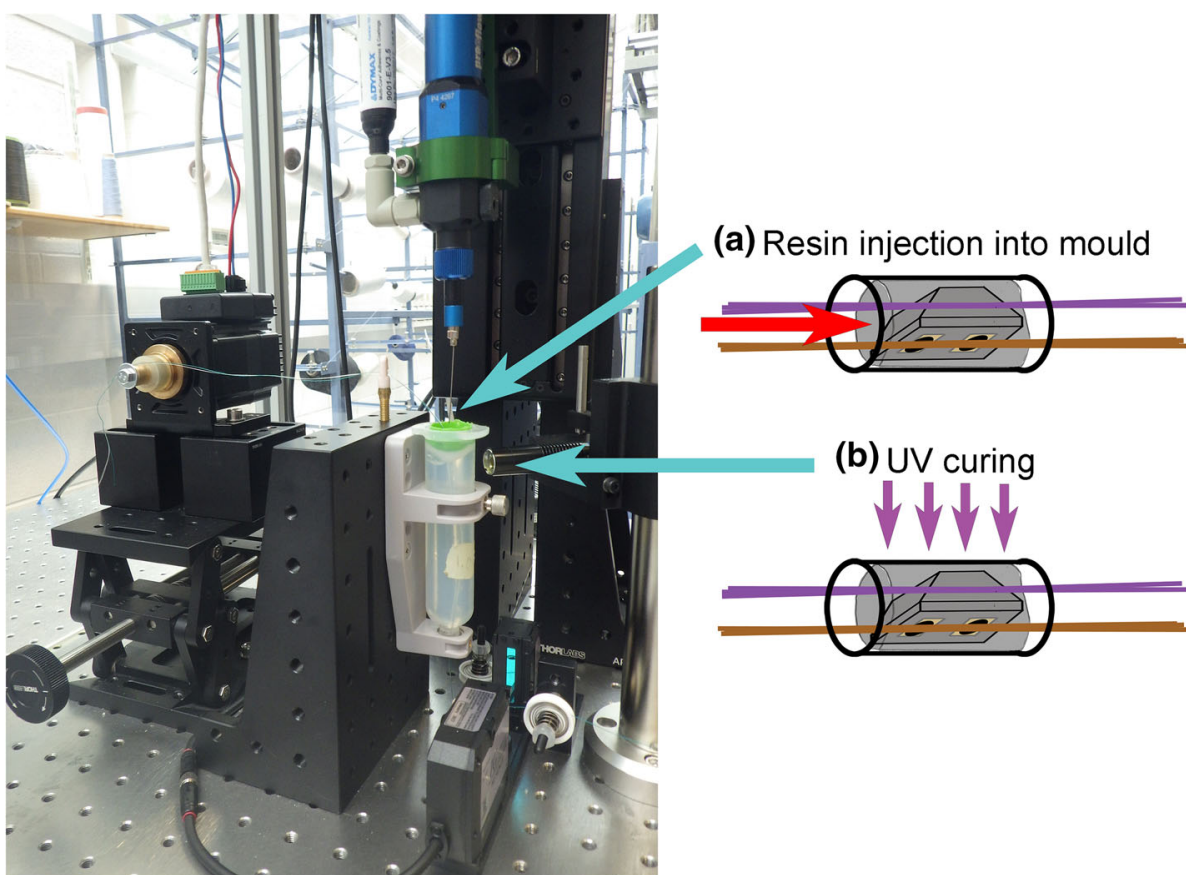




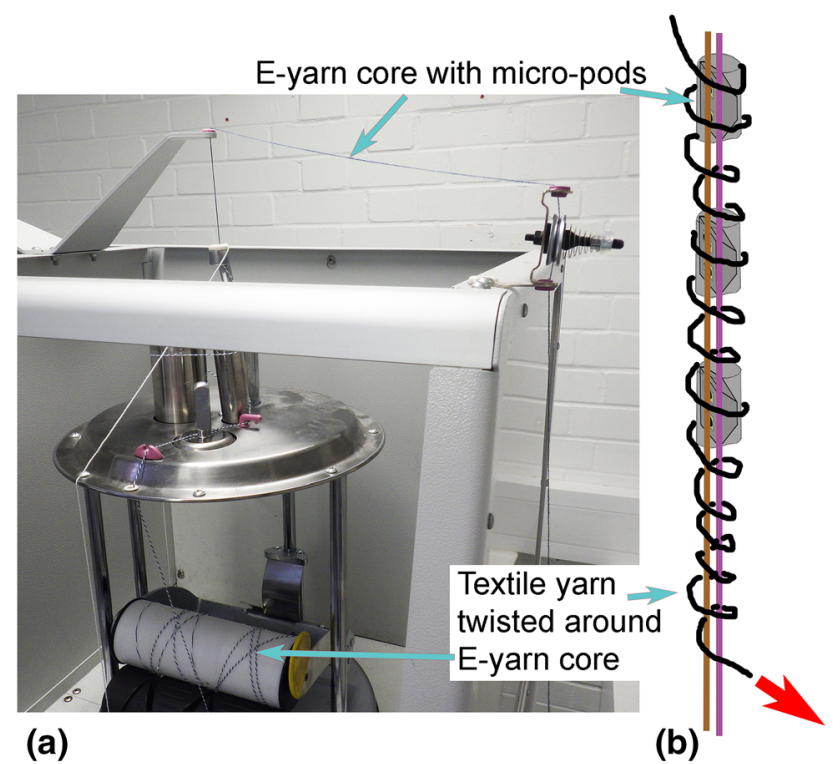

Fig. 7 a The Agteks machine used to twist textile yarn around the E-yarn core, with arrows indicating the location of the E-yarn core and the textile yarns twisted around the E-yarn core; $\mathbf{b}$ the process of wrapping the textile yarns around the E-yarn core

important to cover the entire core, to create an electronic yarn with a textile appearance and feel which could be passed through further textile processing machinery to manufacture garments and other E-textiles. A knitted sheath provided adequate cover for the E-yarn core, but it was necessary to modify the knitting machine, in order to ensure that the E-yarn core remained within the centre of the E-yarn during production. A narrow, central yarn guide was added to ensure that the E-yarn core was positioned accurately in the centre of the E-yarn as the outer textile materials were added (Fig. 8a). A further modification was the addition of six BTSR winding feeders (V14.052.1.0: BTSR, Olgiate Olona, Italy), to control the tension of the six polyester yarns that were being passed through the latch needles of the machine to form the knitted outer sheath.

\subsection{Accuracy of LED placement in the automated manufacturing process}

The accuracy and reliability of the LED placement in the first stage of manufacturing was tested. Ten groups of five LEDs (Kingbright Insertion of E-yarn core into a knitted sheath with addition of packing fibres KPHHS1005SURCK Red LED, 630 nm 1005 (0402), Rectangle Lens SMD package: RS components, Corby, UK) were soldered onto the multi-strand copper wire, with the LEDs within each group being separated by $20 \mathrm{~mm}$. Each LED was $1 \mathrm{~mm}$ long. Measurements were made using a digital calliper (Clarke CM145 Digital Vernier Caliper; Machine Mart Ltd., Nottingham, UK). This tested the accuracy of the placement process using the pick-and-place machine and the wire tensioning equipment.

\subsection{Tensile testing of completed E-yarn and E- yarn components}

The carrier fibre and outer, knitted sheath were expected to add tensile strength to E-yarn in the direction of its longest axis: along the length of the E-yarn. This was assessed by carrying out tensile tests on the E-yarn components, as well as on the completed E-yarn. These were tested on a zwickiline tensile tester (Z2.5: Zwick/Roell, Ulm, Germany) to ASTM E8 (ASTM 2016). This testing standard was designed for use with metallic materials, so the test speed was reduced to $50 \mathrm{~mm} \mathrm{~min}^{-1}$ to assess the performance of the non-elastomeric textile yarns, as well as the copper wire under investigation. The following materials and material combinations were assessed:

1. Copper wire

2. Vectran ${ }^{\mathrm{TM}}$ carrier yarn

3. E-yarn knitted sheath

4. Complete E-yarn

\section{Results}

The automated production process fashioned a flexible yarn of diameter $2 \mathrm{~mm}$ for incorporation into fabrics in subsequent knitting, weaving or embroidery processes. (Note that by using smaller dies, smaller yarn diameters can be produced.) The speed of the production process was increased from 60 to $90 \mathrm{~min}$ per die for the manual, craft process, to 6 min for the prototype, automated process. Figure 9 shows magnified images of LEDs alongside diagrams of the stage in production at which these were produced. Figure 9c shows that the process of twisting textile yarns around copper wire actually led to twisting of the copper wire, with the micropod interrupting the evenness of the twist. Ideally, the textile yarn would be wrapped around the copper. Figure 9d shows a magnified image of a section of completed LED yarn. Figure 10 shows a length of completed LED-yarn.

\subsection{Measuring the accuracy of LED placement}

The accuracy and reliability of the LED placement was tested by measuring the spacing between clusters of five LEDs soldered onto the multi-strand copper wire, with the LEDs within each group to be spaced $20 \mathrm{~mm}$ apart: The results are shown in Fig. 11, below. The standard deviation in the placement was $0.37 \mathrm{~mm}$, corresponding to $1.9 \%$ of the $20 \mathrm{~mm}$ spacing placement. The greatest variation in LED spacing was $0.7 \mathrm{~mm}$ from the target placement point. 


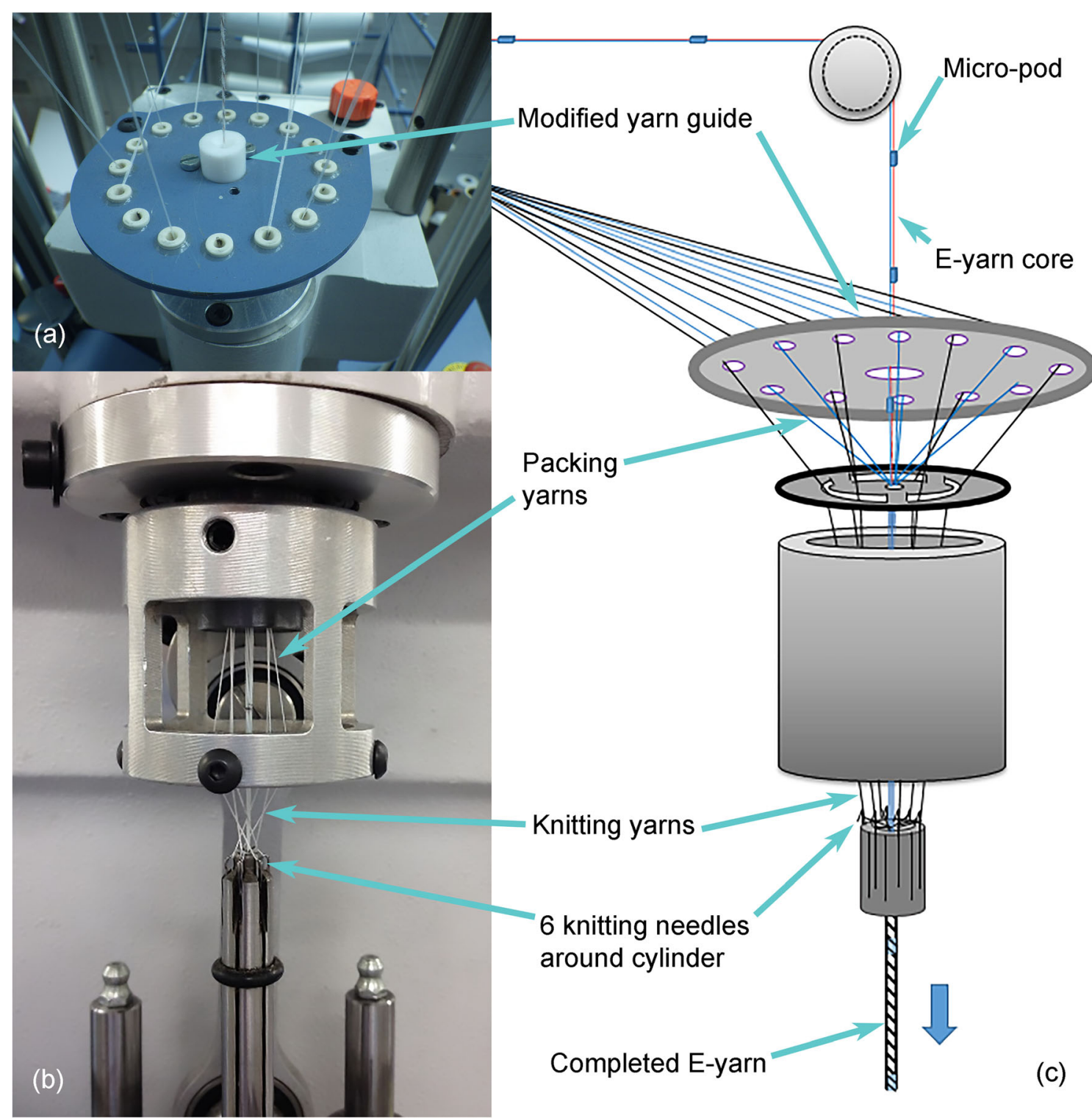

Fig. 8 Insertion of the E-yarn core into a knitted sheath with the addition of packing fibres: a the yarn guide, modified with the addition of a central tube, to ensure that the E-yarn core remains centred during the knitting process; $\mathbf{b}$ the knitting yarns enter the

The LED-spacing depended on the accuracy of the movement of the wheel attached to the stepper motor. The accuracy of LED placement could be improved by optimising the wire feeding system and its interaction with the servo-motor and attached wheel. The focus on the initial system development was on the soldering process, not the movement of the copper wire, and the accuracy of placement will by refined in future prototypes.

\subsection{E-yarn strength}

Figure 12 shows the results of tensile testing of complete E-yarn and E-yarn components. The greatest breaking force knitting needles then the 2-mm internal-diameter cylinder; $\mathbf{c}$ diagram showing the process of placing packing fibres and forming a knitted sheath around an E-yarn core

shown is for the complete E-yarn $(98-111 \mathrm{~N})$. The complete E-yarn was considerably stronger than the copper wire contained in its core, which broke at $2.5-3.5 \mathrm{~N}$, as shown by the dot-dash lines close to the $\mathrm{x}$-axis of the graph. The Vectran ${ }^{\mathrm{TM}}$ breakage is shown by the short, dashed lines on the graph. The Vectran ${ }^{\mathrm{TM}}$ reinforces the construction by having a higher breaking strength than that of the copper, at 23-28 N. The diagonal, dotted lines in the centre of the graph show that the knitted sheath added considerable strength to the construction, breaking at 65-74 N. The elongation of the sheath was similar to that of the copper, with elongation between 17 and $20 \mathrm{~mm}$ for the copper and 19-22 $\mathrm{mm}$ for the E-yarn sheaths. Packing yarns are shown to add a little 

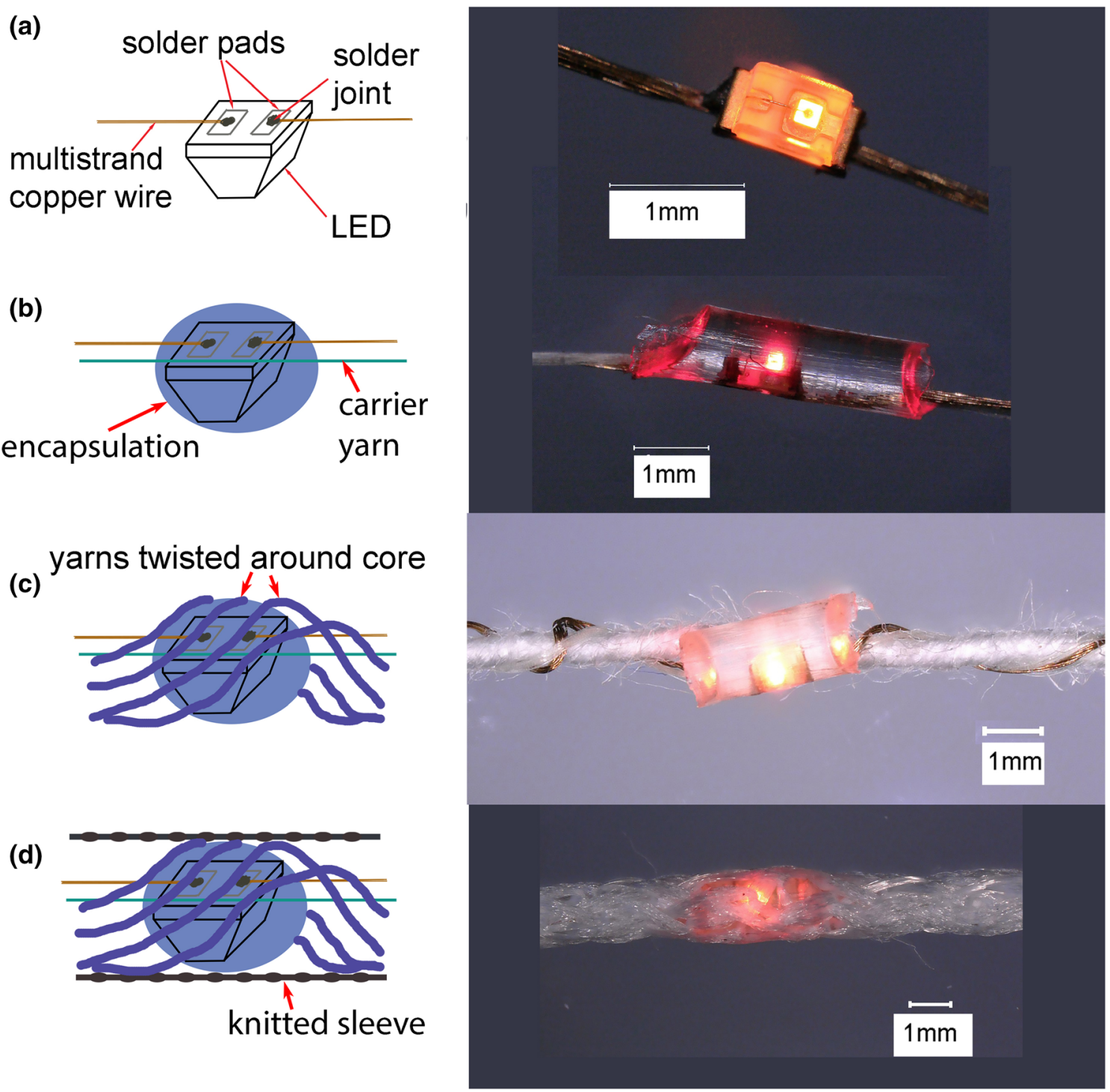

(c)

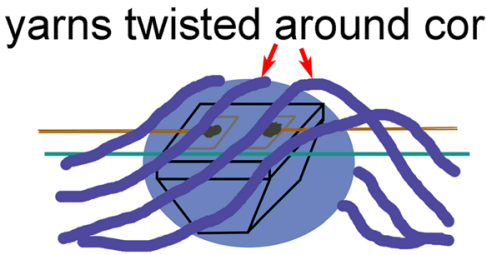

(d)

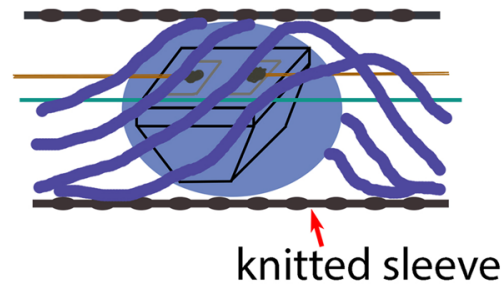

Fig. 9 Diagrams alongside photos showing LED-yarn at each stage of production: a LED soldered to wire with photo at $\times 100$ magnification; $\mathbf{b}$ encapsulated LED on wire with photo at $\times 50$ magnification;

strength to the construction, as shown by the short dot-dash lines showing a breaking strength of $18-21 \mathrm{~N}$, but the elongation is similar to that of the copper wire.

Copper wire and Vectran ${ }^{\mathrm{TM}}$ are put into tension during the manufacturing process of E-yarn, when they are used to pull completed micro-pods from the mould in which they are made. The results for tensile testing of copper wire at the base of Fig. 12 showed that this multi-strand wire elongated by $19-20 \mathrm{~mm}$ over a $100 \mathrm{~mm}$ gauge length before breaking. Vectran ${ }^{\mathrm{TM}}$ exhibits much lower elongation of $0.6-0.9 \mathrm{~mm}$ at the copper breaking force of $3.5 \mathrm{~N}$. The forces applied during E-yarn manufacturing are $1 \%$ of the breaking strain of the copper wire, indicating that the wire is not excessively tensioned during this stage of the c textile yarns twisted around the wire with photo at $\times 30$ magnification; $\mathbf{d}$ completed LED-yarn with photo at $\times 30$ magnification

E-yarn manufacturing process. The Vectran ${ }^{\mathrm{TM}}$ provides additional protection against elongation and breakage of the copper wire during this process, and during the yarn twisting and knitting stages of manufacture.

\subsection{E-yarn core strength}

Textile yarn was twisted around the E-yarn core in stage three of the manufacturing process. The main aim of this was to prevent copper wire from the core from protruding through the knitted sheath of the completed E-yarn. Tensile testing was carried out to ascertain whether the twisted textile yarns contributed to the strength of the E-yarn construction. Figure 13 shows the results of testing of: 
1. Copper wire and Vectran ${ }^{\mathrm{TM}}$ together (the E-yarn core materials).

2. Three strands of cotton yarn (NM 30/1*2 Davidoff: Boyar Textile).

3. Three strands of cotton yarn (NM 30/1*2 Davidoff: Boyar Textile) twisted around copper wire and Vectran $^{\mathrm{TM}}$.

The dashed lines that extend to the right in Fig. 13 show the considerable extension of the cotton yarns before breaking $(27-55 \mathrm{~mm})$. The 3 cotton yarns add some strength to the core when wrapped around copper wire and Vectran $^{\mathrm{TM}}$, as shown by the dotted lines that extend above the solid lines on the left of the figure. The maximum tensile strength of these cores is $33-38 \mathrm{~N}$, which is considerably less than the $98-111 \mathrm{~N}$ strength of the complete E-yarn shown in Fig. 13. The main function of the 3 cotton

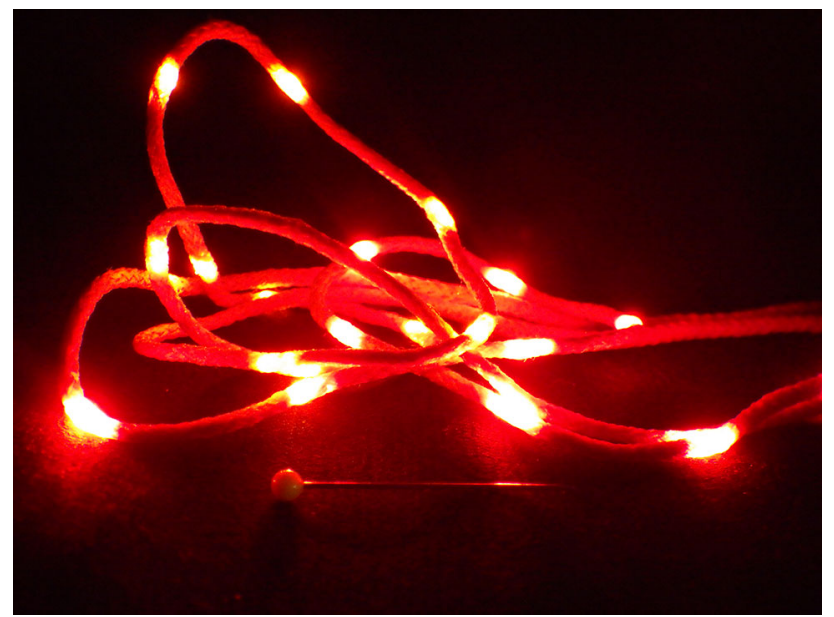

Fig. 10 Completed, illuminated LED yarn, shown next to a $30 \mathrm{~mm}$ long pin

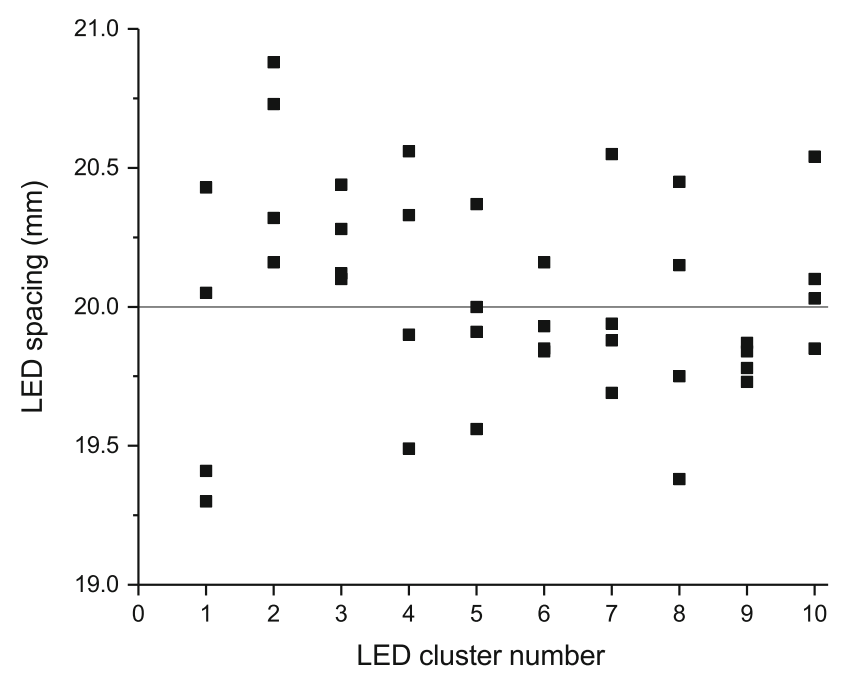

Fig. 11 Variation in placement of LEDs on copper wire at $20 \mathrm{~mm}$ separation using the automated E-yarn production process

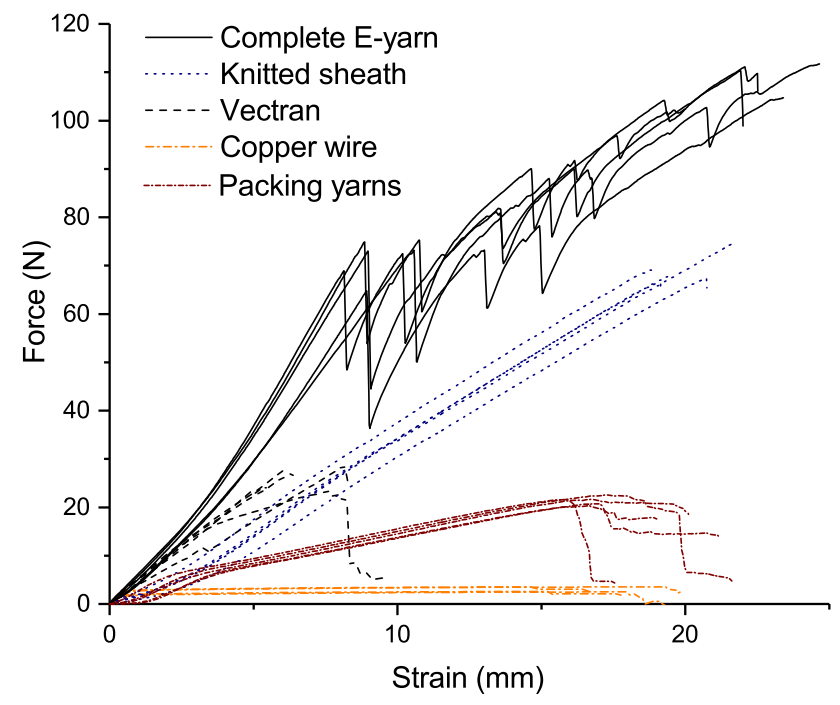

Fig. 12 Tensile test results for complete E-yarn and materials included within E-yarn

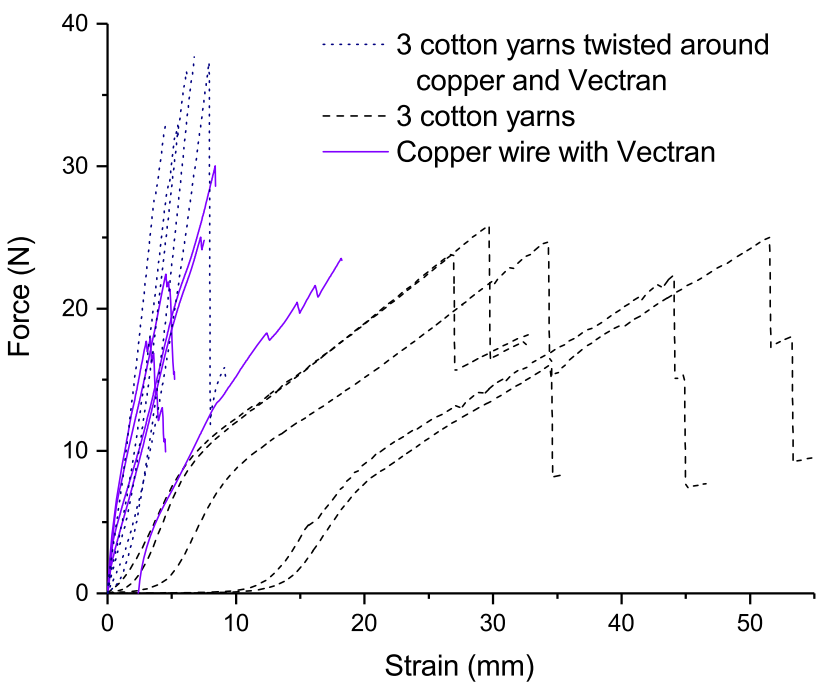

Fig. 13 Tensile test results for materials contained within the core of E-yarn: 3 cotton yarns twisted around copper and Vectran ${ }^{\mathrm{TM}} ; 3$ cotton yarns; copper wire with Vectran ${ }^{\mathrm{TM}}$

yarns was to contain the copper wire within the centre of the E-yarn, so the slightly increased tensile strength is not the main aim.

\section{Further developments}

E-yarns made using the automated manufacturing process have been used in a variety of applications. LED-yarns were attached to stretch fabric to create a carnival costume (Hardy et al. 2018a), showing the feasibility of positioning E-yarns close to the skin, as part of a garment that was required to conform to a dancer's movements. Close contact with the body was also a requirement for E-yarns 
containing thermistors, some of which were manufactured using the semi-automated production process in previous work (Hughes-Riley et al. 2017). A project to create a dress with LED-yarns embroidered onto the surface, further demonstrated that LED-yarns can be integrated into fashionable attire (Bonnie Binary 2018; Hardy et al. 2018c).

Additional developments are required to the automated E-yarn production process described here and to further increase the speed of production. The process involved manual transfer: passing spools of partially-completed E-yarn between stages of the production process. A useful development would be to automate the transfer from one set of machinery to the next. Yarn tension and feed rates will require regulation during the transfers, to ensure optimal delivery to each stage of the production process. The final two stages of E-yarn production (twisting with textile yarns and formation of the knitted sheath) currently operate at a much faster speed than the initial soldering and micro-pod formation stages. A method may be required to hold a slowly-growing volume of copper wire with attached micro-pods, before transfer to the final two manufacturing stages.

The tensile test results demonstrated that the E-yarn sleeve provides much of the strength of the completed E-yarn, with the Vectran ${ }^{\mathrm{TM}}$ carrier yarn providing resistance to breaking during manufacturing. Further development of E-yarn could lead to use of alternative or less materials in the yarn core and outer areas, to optimise strength whilst minimising the amount of different materials used in the E-yarn construction. In particular, a method of using textiles to cover the E-yarn core could obviate the need for the twisting and knitting processes.

Ongoing development of prototype textiles and garments containing E-yarns can now take place in parallel with developments in the E-yarn production process. Further adaptions will enable use insulated copper wire, so that multi-terminal dies can be added into E-yarn using the automated process. The process can also be adapted for incorporation of circuitry populated on Kapton strip into E-yarn ( $\mathrm{Li}$ et al. 2018). Enclosing electronics within the core of yarns could be useful to the process of extracting electronics from electronic textiles, so further investigation of the feasibilities of this can be investigated, to ensure that sustainable recycling and disposal of electronic textiles is at the forefront of development of this technology (McLaren et al. 2017).

\section{Conclusion}

Electronic yarn (E-yarn) contains package dies and conductive elements within a textile casing, creating flexible electronics that can be integrated into textiles that drape and conform. The production of E-yarn was previously a time-consuming craft skill. The automation of the E-yarn production process has resulted in an increase of $1000-1500 \%$ in the speed of production of E-yarn. Accurate placement of dies onto copper wire used in the core of E-yarn was shown to be carried out with an error of $\pm 1.9 \%$. The process can now be developed further; including automation of the movement of partially-completed E-yarn between one set of machinery and the next. This will enable further increases in the speed of production of E-yarns, laying the foundations for a greater adoption of the technology. E-yarns containing sensors and LEDs can be integrated within textiles, including stretch fabrics to be held in close contact with skin. This enables creation of comfortable clothing that can take measurements or provide illumination.

Acknowledgements The authors acknowledge funding from EPSRC grant EP/M015149/1: Novel manufacturing methods for functional electronic textiles. The authors would also like to acknowledge funding from Nottingham Trent University.

The authors would like to thank Dr. Arash Shahidi of Nottingham Trent University for assistance with tensile testing and Jim Boxall for the photos in Fig. 9(a, b, d).

Open Access This article is distributed under the terms of the Creative Commons Attribution 4.0 International License (http://creative commons.org/licenses/by/4.0/), which permits unrestricted use, distribution, and reproduction in any medium, provided you give appropriate credit to the original author(s) and the source, provide a link to the Creative Commons license, and indicate if changes were made.

\section{References}

ASTM (2016) ASTM E8/E8M-16A standard test methods for tension testing of metallic materials. ASTM, West Conshohocken

Bonnie Binary (2018) Red Dress featuring illuminated electronic yarn-Bonnie Binary. http://www.bonniebinary.co.uk/portfolio/ red-dress/. Accessed 20 Mar 2018

Dias T (2005) Operative devices in yarns. Patent GB2426255B1, EP1882059B1

Dias T (2016) Electronic functional yarn. Patent GB2529900B1

Hardy D, Moneta A, Sakalyte V et al (2018a) Engineering a costume for performance using illuminated LED-yarns. Fibers 6:35. https://doi.org/10.3390/fib6020035

Hardy DA, Anastasopoulos I, Nashed M-N et al (2018b) An automated process for inclusion of package dies and circuitry within a textile yarn. In: 2018 Symposium on Design, Test, Integration \& Packaging of MEMS and MOEMS (DTIP). IEEE, New York

Hardy DA, Oliveira C, Nashed M-N, Dias T (2018c) Textiles illuminated with electronic yarn. In: Poster session presented at IDTechEx 2018. Berlin

Hayward J (2017) E-Textiles 2017-2027: technologies, markets, players. In: IDTechEx. https://www.idtechex.com/research/ reports/e-textiles-2017-2027-technologies-markets-players000522.asp. Accessed 8 Feb 2018 
Hughes-Riley T, Dias T (2018a) Developing an acoustic sensing yarn for health surveillance in a military setting. Sensors 18:1590. https://doi.org/10.3390/s18051590

Hughes-Riley T, Dias T (2018b) The development of acoustic and vibration sensing yarns for health surveillance. In: 25th international congress on sound and vibration (ICSV25). Hiroshima, Japan

Hughes-Riley T, Lugoda P, Dias T et al (2017) A study of thermistor performance within a textile structure. Sensors 17:1804. https:// doi.org/10.3390/s17081804

Hughes-Riley T, Dias T, Cork C (2018) A historical review of the development of electronic textiles. Fibers 6:34. https://doi.org/ 10.3390/fib6020034

LaDou J (2006) Printed circuit board industry. Int J Hyg Environ Health 209:211-219. https://doi.org/10.1016/j.ijheh.2006.02.001

Li J, Shrivastava P, Gao Z, Zhang H-C (2004) Printed circuit board recycling: a state-of-the-art survey. IEEE Trans Electron Packag Manuf 27:33-42. https://doi.org/10.1109/TEPM.2004.830501

Li M, Tudor J, Torah R, Beeby S (2018) Stress analysis and optimization of a flip chip on flex electronic packaging method for functional electronic textiles. IEEE Trans Compon Packag Manuf Technol 8:186-194. https://doi.org/10.1109/TCPMT. 2017.2780626
Lu SC-Y, Liu A (2012) Abductive reasoning for design synthesis. CIRP Ann Manuf Technol 61:143-146. https://doi.org/10.1016/j. cirp.2012.03.062

Lugoda P, Hughes-Riley T, Oliveira C et al (2018) Developing novel temperature sensing garments for health monitoring applications. Fibers 6:46. https://doi.org/10.3390/fib6030046

McLaren A, Hardy DA, Hughes-Riley T (2017) Electronic textiles and product lifetimes: exploring design strategies for product longevity. In: Bakker CA, Mugge R (eds) PLATE: product lifetimes and the environment. IOS Press, Delft

Rathnayake AS (2015) Development of the core technology for the creation of electronically-active, smart yarn. Nottingham Trent University, Nottingham

Siegel AC, Phillips ST, Dickey MD et al (2010) Foldable printed circuit boards on paper substrates. Adv Funct Mater 20:28-35. https://doi.org/10.1002/adfm.200901363

Tomico O, Hallnäs L, Liang R-H, Wensveen SAG (2017) Towards a next wave of wearable and fashionable interactions. Int J Des $11: 1-6$

Publisher's Note Springer Nature remains neutral with regard to jurisdictional claims in published maps and institutional affiliations. 